\title{
On the Performance of Power Splitting-Based SWIPT in Self-Energy Recycling Full-Duplex Relaying Networks
}

\author{
I. W. G. da Silva*, D. P. M. Osorio ${ }^{\dagger}$, E. E. B. Olivo ${ }^{\ddagger}$, O. L. A. López ${ }^{\dagger}$, H. Alves ${ }^{\dagger}$, and M. Latva-aho ${ }^{\dagger}$ \\ *Department of Electrical Engineering, Federal University of São Carlos, São Carlos, Brazil \\ ${ }^{\dagger} 6 \mathrm{G}$ Flagship, University of Oulu, Finland \\ ${ }^{\ddagger}$ São Paulo State University (UNESP), Campus of São João da Boa Vista, Brazil \\ E-mails: isabella@estudante.ufscar.br,diana.moyaosorio@oulu.fi, edgar.olivo@unesp.br,onel.alcarazlopez@oulu.fi, hirley.alves@oulu.fi, matti.latva-aho@oulu.fi
}

\begin{abstract}
This paper investigates the outage performance of a cooperative relaying network, where the relay node is considered to be an energy-constrained device so that a power splitting-based simultaneous wireless information and power transfer scheme is employed. The relay is considered to operate in full-duplex (FD) mode so that both energy recycling and information decoding can be performed. For this purpose, the relay is assumed to be provided with two batteries which switch between the power supplying mode and charging mode at each transmission block. In particular, we assume that the self-interference inherent to FD mode is not completely suppressed; it is subject only to passive interference cancellation for self-energy recycling, while it is subject to both passive and active cancellation for information decoding. We derive a tight closed-form approximation to the outage probability for the considered FD mode-based scheme, as well as for the HD mode-based counterpart. We validate the obtained expressions via Monte Carlo simulations. The impact of self-energy recycling in FD mode on the system performance is assessed.
\end{abstract}

\section{INTRODUCTION}

5G uses cases are broadly classified into three main categories [1]: enhanced Mobile Broadband (eMBB), UltraReliable and Low-Latency Communications (URLLC), and massive Machine-Type Communications (mMTC). These three categories account for a myriad of highly diverse applications from different industry sectors. Particularly, mMTC focuses on scenarios with massive deployments of devices as those of Internet of Things (IoT) [2]. Therefore, network overload as well as energy consumption to keep thousands of devices connected is one of mMTC's greatest challenges.

The development of clean technologies that make the communication network self-sufficient is of utmost importance [3]. One of the techniques that presents promising results in the study of self-supporting mobile communication networks is energy harvesting (EH) [4]. EH consists in a process of converting energy from external sources (such as wind, sun, vibration, etc.) in electrical energy to be stored and used to power a device or recharge batteries. However, these kinds of sources cannot be controlled and not always are available for immediate use. To overcome such difficulties, an option that has attracted great attention is wireless energy transfer (WET) which is based on the use of radio frequency (RF) waves to perform EH [5].
In this reason, the simultaneous wireless information and power transfer (SWIPT) can result in notable gains in energy consumption, spectral efficiency, interference control and transmission delay [6]. However, in practice, it is not possible to perform EH and decode the information at the same time. To perform SWIPT, the signal must be separated into two different parts, one for energy harvesting and another for information. Different techniques have been proposed in the literature in order to implement this separation, namely Power Splitting (PS) [7], [8]; Time Switching (TS) [9]; Antenna Switching [10]; and Spatial Switching [11]. In addition, several SWIPT scenarios have been studied with the aim of improving the efficiency of the system, addressing different strategies, e.g., Non-Orthogonal Multiple Access (NOMA) [12], Multiple-Input Multiple-Output (MIMO) systems [13] and relaying techniques.

In particular, relaying techniques are intended to achieve spatial diversity without the need of multiple antennas at the terminals [14], thus achieving great benefits in terms of capacity and reliability. The most commonly used protocols for relaying networks are Amplify-and-Foward (AF) and Decodeand-Foward (DF). Thus, SWIPT schemes have been investigated in order to power energy constrained devices acting as relays in different schemes [15], [16]. For instance, the case of untrusted relays was studied in [15], and full-duplex (FD) relaying was studied in [16].

FD consists on transmitting and receiving data simultaneously, resulting in significant gains compared to half-duplex (HD). Commonly, FD is accomplished by using two or more antennas. However, in practice, this technique shows major self-interference (SI) problems [17]. By using interference cancellation techniques, SI can be highly attenuated; nevertheless, it cannot be completely removed, making FD performance limited by a residual self-interference (RSI). This scenario has gained a lot attention and has been widely studied in [18], [19].

In this paper, we investigate the outage performance of a relay-based cooperative network, where the relay node is considered to be an energy-constrained device, thus a power splitting-based simultaneous wireless energy and power transfer scheme is used in order to the relay have energy to perform the retransmission of information. We evaluate and compare both cases, when the relay is a FD device and the HD relay in 
order to verify the impact of self-energy recycling in FD mode. We derive tight closed-form approximate expressions for the outage probability of both cases, HD and FD and validate them via Monte Carlo simulations.

\section{SySTEM MODEL}

\section{A. Full-duplex mode}

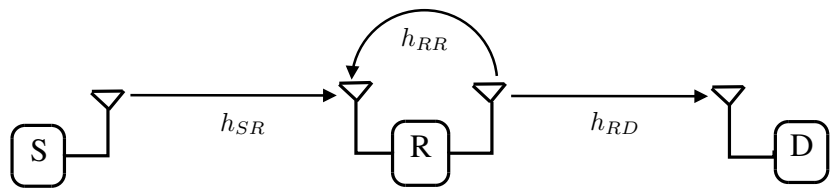

Fig. 1. System Model

Fig. 1 illustrates a wireless energy transfer network where one source $(\mathrm{S})$ intends to send its information to the destination (D) with the help of a DF relay (R). The relay is assumed to be energy-constrained such that it has to harvest energy of the RF signals received from $\mathrm{S}$ to forward information. All terminals are equipped with a single antenna except for $\mathrm{R}$, which is equipped with one pair of transmit and receive antennas in order to operate in FD mode. The direct link between $\mathrm{S}$ and $\mathrm{D}$ is assumed to be severely attenuated, such that the communication is only feasible through $\mathrm{R}$.

Additionally, all links are considered to undergo independent Rayleigh block fading, as well as additive white gaussian noise (AWGN) with average power $N_{0}$. Accordingly, (i) $h_{S R}$ and $h_{R D}$ are the channels coefficients for the links S-R and R-D, respectively and are assumed to be independent circularly-symmetric Gaussian random variables $\mathcal{C N}\left(0, d_{m}^{-\varphi}\right)$, $m \in\{S R, R D\}$; (ii) $d_{m}$ with $m \in\{S R, R D\}$ are the distances between the corresponding pairs of transceivers and (iii) $\varphi$ is the pahtloss exponent.

At the relay, by considering the FD mode operation, the channel coefficient of RSI at the receive antenna after passive interference cancellation (self-energy recycling channel), and that obtained after active (analog and digital) interference cancellation (AIC) are denoted by $h_{\mathrm{RR}}^{\mathrm{E}}$ and $h_{\mathrm{RR}}^{\mathrm{I}}$, respectively. Assuming that the line-of-sight (LOS) component of the signal is completely cancelled after the passive IC process, $h_{E H}$ is modeled as a Rayleigh fading channel. Due to the different atenuation after the active IC, $h_{\mathrm{RR}}^{\mathrm{I}}$ is modeled as another Rayleigh fading channel [20].

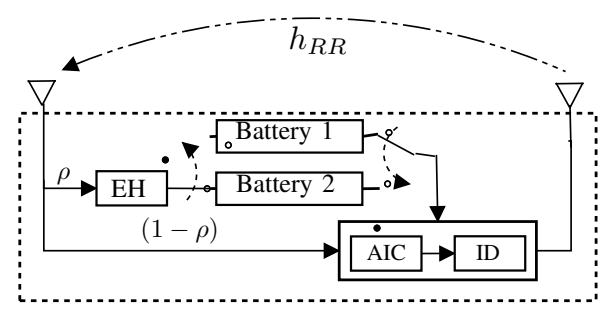

Fig. 2. Block Diagram of the considered FDR node

As in [21], the proposed virtual harvest-use model deploys a battery group consisting of two rechargeable batteries, as depicted in Fig. 2. It is assumed that each battery contains enough redundant energy initially. The two batteries are activated for $\mathrm{EH}$ and power supplying alternately in a time switching manner with each block of transmission denoted by $T$ and divided equally into two time slots.

In each time slot, The power of the relay-received signal is split for EH and information decoding (ID), after active IC, according to the proportion $\rho:(1-\rho)$, where $\rho \in(0,1)$ is the PS ratio. Under these considerations, the input signal at the energy harvest receiver can be written as

$$
y_{\mathrm{EH}}=\sqrt{\rho}\left(\sqrt{P_{S}} h_{\mathrm{SR}} s_{s}(t)+\sqrt{P_{R}} h_{\mathrm{RR}}^{\mathrm{E}} s_{r}(t)\right)
$$

where $P_{S}$ and $P_{R}$ are the source and relay transmit powers, respectively. $s_{s}(t)$ is the signal transmitted from $\mathrm{S}$ and $s_{r}(t)$ is the relay transmit signal. For notation simplicity, consider $g_{i} \triangleq\left|h_{i}\right|^{2}$ and $\Omega_{i}=E\left(\left|h_{i}\right|^{2}\right)$, for $i \in\{S R, I D, E H, R D\}$ as the channel gains and the average channel gain, respectively. This way, at the end of a time slot, the harvested energy at the relay can be expressed as

$$
E_{H}=\eta \rho\left(P_{S} g_{\mathrm{SR}}+P_{R} g_{\mathrm{RR}}^{\mathrm{E}}\right) \frac{T}{2}
$$

where $\eta \in(0,1)$ is the energy conversion and battery discharging utilizing efficiency factor. Simultaneously, the transmission is powered by the battery that is not switched by the EH. Considering $E_{H}$ as the amount of energy available to the transmission, $P_{R}$ can be written as

$$
P_{R}=\frac{E_{H}}{T / 2} \triangleq \eta \rho\left(P_{S} g_{\mathrm{SR}}+P_{R} g_{\mathrm{RR}}^{\mathrm{E}}\right) \triangleq \frac{\eta \rho P_{S} g_{\mathrm{SR}}}{\left(1-\eta \rho g_{\mathrm{RR}}^{\mathrm{E}}\right)}
$$

Due to passive IC, such as antenna cancellation, $h_{\mathrm{RR}}^{\mathrm{E}}$ is less than 1, so (3) is always positive. With the DF protocol, assuming the correct decoding of the signal, the relay transmit signal can be written as $s_{r}(t)=s_{s}(t-k)$, where $k$ is the processing delay at the relay. Therefore, the received signal at the information decoding (ID) receiver and at the destination are respectively given by

$y_{R}=\sqrt{(1-\rho) P_{S}} h_{\mathrm{SR}} s_{s}(t)+\sqrt{(1-\rho) P_{R}} h_{\mathrm{RR}}^{\mathrm{I}} s_{s}(t-k)+n_{r}(t)$

$y_{D}=\sqrt{P_{R}} h_{\mathrm{RD}} s(t-k)+n_{D}$

Where $n_{r}$ and $n_{D}$ are the noise components at $\mathrm{R}$ and $\mathrm{D}$, respectively. Based on (4) and (5), the SINR at the relay and at the destination can be expressed as

$$
\begin{aligned}
\gamma_{R} & =\frac{(1-\rho) P_{S} g_{\mathrm{SR}}}{\frac{(1-\rho) \eta \rho P_{S} g_{\mathrm{SR}} g_{\mathrm{RR}}^{\mathrm{I}}}{1-\eta \rho g_{\mathrm{RR}}^{\mathrm{E}}}+N_{0}} \\
\gamma_{D} & =\frac{P_{R} g_{\mathrm{RD}}}{N_{0}}
\end{aligned}
$$

B. Half duplex mode

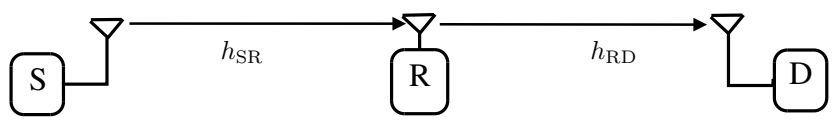

Fig. 3. System Model 


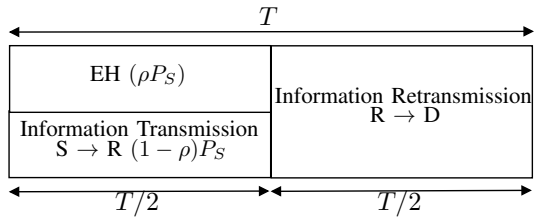

Fig. 4. Block Diagram of the half-duplex relay transmission scheme

Fig. 3 illustrates the wireless energy transfer network with HD relay. Furthermore, as shown in Fig. 4, the transmission is split into two intervals. First, the source sends a signal to $\mathrm{R}$ that is divided to $\mathrm{EH}$ and ID according to the proportion $\rho:(1-\rho)$, where $\rho \in(0,1)$ is the PS ratio. Similar to the analysis to obtain (2), the harvested energy after the end of a time slot and the respective relay transmission power can be expressed as

$$
\begin{aligned}
& E_{H_{\mathrm{HD}}}=\eta \rho P_{S} g_{\mathrm{SR}} \frac{T}{2} \\
& P_{R_{\mathrm{HD}}}=\frac{E_{H_{\mathrm{HD}}}}{T / 2} \triangleq \eta \rho P_{S} g_{\mathrm{SR}}
\end{aligned}
$$

This way, the instantaneous SINR at the relay and at the destination can be, respectively, written as $\gamma_{R}=(1-\rho) P_{S} g_{\mathrm{SR}} / N_{0}$ and $\gamma_{D}=P_{R} g_{\mathrm{RD}} / N_{0}$

\section{Outage Probability}

\section{A. Full duplex mode}

By definition, the system is in outage if the instantaneous end-to-end SINR is less than an given threshold $\tau$. By considering the DF relaying protocol, the outage probability is given by

$$
P_{\text {out }}=\operatorname{Pr}\left[\min \left\{\gamma_{R}, \gamma_{D}\right\}<\tau\right]=1-\underbrace{\operatorname{Pr}\left[\gamma_{R}>\tau, \gamma_{D}>\tau\right]}_{\Theta}
$$

Note that, as in [22], $\gamma_{R}$ and $\gamma_{D}$ are correlated random variables. That means that the SNR at D depends on what happens on the $S \rightarrow R$ link, hence the joint CDF presented in (10) cannot be analysed as the product between the marginals CDFs, i.e., $F_{\gamma_{R}, \gamma_{D}}(\cdot, \cdot) \neq F_{\gamma_{R}}(\cdot) F_{\gamma_{D}}(\cdot)$. Therefore, the term $\Theta$ can be expressed as

$$
\begin{array}{r}
\Theta_{\mathrm{FD}}=\int_{0}^{\eta \rho} \int_{\frac{\tau}{\gamma_{P}-\gamma_{P} \rho}}^{\infty} \int_{\frac{\tau-\eta \rho \tau g_{\mathrm{RR}}^{\mathrm{E}}}{\gamma_{P} \eta \rho g_{\mathrm{SR}}}}^{\infty} F_{\mathrm{ID}}\left(g_{\mathrm{RR}}^{\mathrm{I}}\right) f_{\mathrm{RD}}\left(g_{\mathrm{RD}}\right) f_{\mathrm{SR}}\left(g_{\mathrm{SR}}\right) \\
f_{\mathrm{EH}}\left(g_{\mathrm{RR}}^{\mathrm{E}}\right) d g_{\mathrm{RD}} d g_{\mathrm{SR}} d g_{\mathrm{RR}}^{\mathrm{E}}
\end{array}
$$

All channels are subjected to Rayleigh fading and their corresponding power gains are exponentially distributed. Under these considerations, the probability density functions $f_{x}(\cdot)$ with $X \in(S R, R D, E H)$ can be written as $f_{\mathrm{X}}\left(g_{\mathrm{X}}\right)=$ $e^{-\frac{g_{\mathrm{X}}}{\Omega_{\mathrm{X}}}} / \Omega_{\mathrm{x}}$ and the $\operatorname{CDF} F_{I D}(\cdot)$ is given by

$$
F_{\mathrm{ID}}\left(g_{\mathrm{RR}}^{\mathrm{I}}\right)=1-\exp \left(\frac{\left(\eta \rho g_{\mathrm{RR}}^{\mathrm{E}}-1\right)\left(\gamma_{P}(\rho-1) g_{\mathrm{SR}}+\tau\right)}{\gamma_{P} \eta(\rho-1) \rho \tau \Omega_{\mathrm{DI}} g_{\mathrm{SR}}}\right)
$$

First, solving (11) for $g_{R D}$ results in

$$
\begin{aligned}
\int_{0}^{\eta \rho} \int_{\frac{\tau}{\gamma_{P}-\gamma_{P} \rho}}^{\infty} & -\frac{e^{\left(\frac{\tau\left(\eta \rho g_{\mathrm{RR}}^{\mathrm{E}}-1\right)}{\gamma_{P} \eta \rho g_{\mathrm{SR}} \Omega_{\mathrm{RD}}}-\frac{g_{\mathrm{RR}}^{\mathrm{E}}}{\Omega_{\mathrm{RR}}^{\mathrm{E}}}-\frac{g_{\mathrm{SR}}}{\Omega_{\mathrm{SR}}}\right)}}{\Omega_{\mathrm{RR}}^{\mathrm{E}} \Omega_{\mathrm{SR}}} \\
& \times\left(e^{\left(\frac{\left(\eta \rho g_{\mathrm{RR}}^{\mathrm{E}}-1\right)\left(\gamma_{P}(\rho-1) g_{\mathrm{SR}}+\tau\right)}{\gamma_{P} \eta(\rho-1) \rho \tau \Omega_{\mathrm{RR}}^{\mathrm{I}} g_{\mathrm{SR}}}\right)}-1\right) d g_{\mathrm{SR}} d g_{\mathrm{RR}}^{\mathrm{E}}
\end{aligned}
$$

Since there is no direct solution of (13) for the $g_{S R}$ variable, it was divided into two more integrals as shown below

$$
\begin{aligned}
\sum_{n=0}^{\infty} \int_{\frac{\tau}{\gamma_{P}-\gamma_{P} \rho}}^{\infty} & \frac{e^{-\frac{g_{\mathrm{RR}}^{\mathrm{E}}}{\Omega_{\mathrm{RR}}^{\mathrm{E}}}-\frac{g_{\mathrm{SR}}}{\Omega_{\mathrm{SR}}}}\left(\frac{\tau\left(\eta \rho g_{\mathrm{RR}}^{\mathrm{E}}-1\right)}{\gamma_{P} \eta \rho g_{\mathrm{SR}} \Omega_{\mathrm{RD}}}\right) n}{n ! \Omega_{\mathrm{RR}}^{\mathrm{EH}} \Omega_{\mathrm{SR}}} d g_{\mathrm{SR}} \\
\sum_{n=0}^{\infty} \int_{\frac{\tau}{\gamma_{P}-\gamma_{P} \rho}}^{\infty} & -\frac{e^{\frac{\eta \rho g_{\mathrm{RR}}^{\mathrm{E}}-1}{\eta \rho \tau \Omega_{\mathrm{RR}}^{1}}-\frac{g_{\mathrm{RR}}^{\mathrm{E}}}{\Omega_{\mathrm{RR}}^{\mathrm{E}}}-\frac{g_{\mathrm{SR}}}{\Omega_{\mathrm{SR}}}}}{n ! \Omega_{\mathrm{RR}}^{\mathrm{E}} \Omega_{\mathrm{SR}}} \\
& \times\left(\frac{\left(\eta \rho g_{\mathrm{RR}}^{\mathrm{E}}-1\right)\left((\rho-1) \tau \Omega_{\mathrm{RR}}^{\mathrm{I}}+\Omega_{\mathrm{RD}}\right)}{\gamma_{P} \eta(\rho-1) \rho g_{\mathrm{SR}} \Omega_{\mathrm{RR}}^{\mathrm{I}} \Omega_{\mathrm{RD}}}\right)^{n} d g_{\mathrm{SR}}
\end{aligned}
$$

Note that beyond the division into the two integrals, it was necessary to expand some of the exponencial functions into a series according to the equation $e^{x}=\sum_{n=0}^{\infty} \frac{x^{n}}{n !}$. Solving (14) and (15) we obtain, respectively,

$$
\begin{aligned}
& \sum_{n=0}^{\infty} \frac{e^{-\frac{g_{\mathrm{RR}}^{\mathrm{E}}}{\Omega_{\mathrm{RR}}^{\mathrm{E}}} \Gamma\left(1-n, \frac{\tau}{\left(\gamma_{P}-\gamma_{P} \rho\right) \Omega_{\mathrm{SR}}}\right)\left(\frac{\gamma_{P} \eta \rho \Omega_{\mathrm{RD}} \Omega_{\mathrm{SR}}}{\tau}\right)-n}}{n ! \Omega_{\mathrm{RR}}^{\mathrm{E}}\left(\eta \rho g_{\mathrm{RR}}^{\mathrm{E}}-1\right)^{-n}} \\
& \sum_{n=0}^{\infty} \frac{\tau e^{\frac{\eta \rho g_{\mathrm{RR}}^{\mathrm{E}}-1}{\eta \rho \tau \Omega_{\mathrm{DI}}}-\frac{g_{\mathrm{R}}^{\mathrm{E}}}{\Omega_{\mathrm{RR}}^{\mathrm{E}}}} E_{n}\left(\frac{\tau}{\gamma_{P} \Omega_{\mathrm{SR}}-\gamma_{P} \rho \Omega_{\mathrm{SR}}}\right)}{\gamma_{P}(\rho-1) n ! \Omega_{\mathrm{RR}}^{\mathrm{E}} \Omega_{\mathrm{SR}}} \\
& \times\left(-\frac{\left(\eta \rho g_{\mathrm{RR}}^{\mathrm{E}}-1\right)\left((\rho-1) \tau \Omega_{\mathrm{DI}}+\Omega_{\mathrm{RD}}\right)}{\eta \rho \tau \Omega_{\mathrm{DI}} \Omega_{\mathrm{RD}}}\right) n
\end{aligned}
$$

Thus, by integrating (16) and (17) for $g_{E H}, \Theta$ is closely approximated by (18) at the top of next page. On this expression, $E_{n}(\cdot)$ is the exponencial integral E function [23], $\Gamma(\cdot)$ is the gamma function [24, Eq. 8324] and $\Gamma(\cdot, \cdot)$ is the incomplete Gamma function [24, Eq. 8350.2].

\section{B. Half duplex mode}

Considering the outage probability expression defined in (10) and the half duplex mode, the term $\Theta_{\mathrm{HD}}$ is given by

$$
\Theta_{\mathrm{HD}}=\int_{\frac{\tau}{\gamma_{P}-\gamma_{P} \rho}}^{\infty}\left(1-F_{\mathrm{RD}}\left(g_{\mathrm{RD}}\right)\right) f_{\mathrm{SR}}\left(g_{\mathrm{SR}}\right) d g_{\mathrm{SR}}
$$

Where $\left.F_{\mathrm{RD}}\left(g_{\mathrm{RD}}\right)\right)=1-e^{-\frac{\tau}{\gamma_{P} \eta \rho g_{\mathrm{SR}} \Omega_{\mathrm{RD}}}}$. Under these considerations, $\Theta_{\mathrm{HD}}$ is approximated as

$$
\Theta_{\mathrm{HD}} \approx \sum_{n=0}^{N} \frac{\cos (n \pi) \Gamma\left(1-n, \frac{\tau}{\left(\gamma_{P}-\gamma_{P} \rho\right) \Omega_{\mathrm{SR}}}\right)(\tau)^{n}}{n !\left(\gamma_{P} \eta \rho \Omega_{\mathrm{SR}} \Omega_{\mathrm{RD}}\right)^{n}}
$$




$$
\begin{array}{r}
\Theta \approx \sum_{n=0}^{N} \frac{\tau^{2} \Omega_{\mathrm{RR}}^{\mathrm{I}} \Omega_{\mathrm{RD}}^{-n} e^{-\frac{1}{\eta \rho \Omega_{\mathrm{RR}}^{\mathrm{E}}}}\left(\Omega_{\mathrm{RR}}^{\mathrm{E}}\left((\rho-1) \tau \Omega_{\mathrm{RR}}^{\mathrm{I}}+\Omega_{\mathrm{RD}}\right)\right)^{n} \mathrm{E}_{n}\left(\frac{\tau}{\gamma_{P} \Omega_{\mathrm{SR}}-\gamma_{P} \rho \Omega_{\mathrm{SR}}}\right)\left(\Gamma(n+1)-\Gamma\left(n+1, \frac{\Omega_{\mathrm{RR}}^{\mathrm{E}}-\tau \Omega_{\mathrm{RR}}^{\mathrm{I}}}{\eta \rho \tau \Omega_{\mathrm{RR}}^{\mathrm{E}} \Omega_{\mathrm{RR}}^{\mathrm{R}}}\right)\right)}{\left(\Omega_{\mathrm{RR}}^{\mathrm{E}}-\tau \Omega_{\mathrm{RR}}^{\mathrm{I}}\right)^{n+1} \gamma_{P}(\rho-1) n ! \Omega_{\mathrm{SR}}} \\
+\sum_{n=0}^{N}-\frac{e^{-\frac{1}{\eta \rho \Omega_{\mathrm{RR}}^{\mathrm{E}}}}\left(\Gamma(n+1)-\Gamma\left(n+1,-\frac{1}{\eta \rho \Omega_{\mathrm{RR}}^{\mathrm{E}}}\right)\right)\left(\frac{\tau \Omega_{\mathrm{RR}}^{\mathrm{E}}}{\gamma_{P} \Omega_{\mathrm{RD}} \Omega_{\mathrm{SR}}}\right)^{n} \Gamma\left(1-n, \frac{\tau}{\left(\gamma_{P}-\gamma_{P} \rho\right) \Omega_{\mathrm{SR}}}\right)}{n !}
\end{array}
$$

\section{NumericAl Results}

In this section, the analytical expressions derived in the previouly section are evaluated for illustrative scenarios. We also provide Monte Carlo simulations to corroborate our analysis. Let us consider a linear network topology in which the distances between $\mathrm{S}$ and $\mathrm{R}, \mathrm{R}$ and $\mathrm{D}$ and between $\mathrm{S}$ and $\mathrm{D}$ are, respectively, set to $d_{\mathrm{SR}}=0.5, d_{\mathrm{RD}}=0.5$ and $d_{\mathrm{SD}}=1$. In addiction, 5 is set as a good approximation of the $N$ factor in (18) and (20). Moreover, we assume a pathloss exponent $\varphi=4$, an SNR threshold $\tau=2^{2 \mathcal{R}}-1$ for the HD relay mode and $\tau=2^{\mathcal{R}}-1$ for the FDR mode with $\mathcal{R}=1$ and the EH efficiency factor to $\eta=1$.

Fig. 5 shows the outage probability as a function of the transmit SNR, $\gamma_{P}$, for different combinations of the RSI links, $\Omega_{\mathrm{RR}}^{\mathrm{I}}$ and $\Omega_{\mathrm{RR}}^{\mathrm{E}}$, considering a power splitting factor, $\rho=0.5$ and a comparison with the scenario in HD mode. The analytical expressions are corroborated via Monte Carlo simulations. It is observed that both analytical expressions perfectly matches the Monte Carlo simulations. Notice that the self-energy recycling average channel gain, $\Omega_{\mathrm{RR}}^{\mathrm{E}}$ is more influent on the performance of the system than reducing the RSI modeled by the channel $g_{\mathrm{RR}}^{\mathrm{I}}$. Also notice that, the self-energy recycling proves to be detrimental to the system performance as it increases the interference do to RSI at the relay, thus by increasing its value a loss in performance is observed. It is also observed that due to the influence of the RSI for higher values of $\gamma_{P}$, for $\gamma_{P}$ greater than $30 \mathrm{~dB}$, the HD mode obtain better performance.

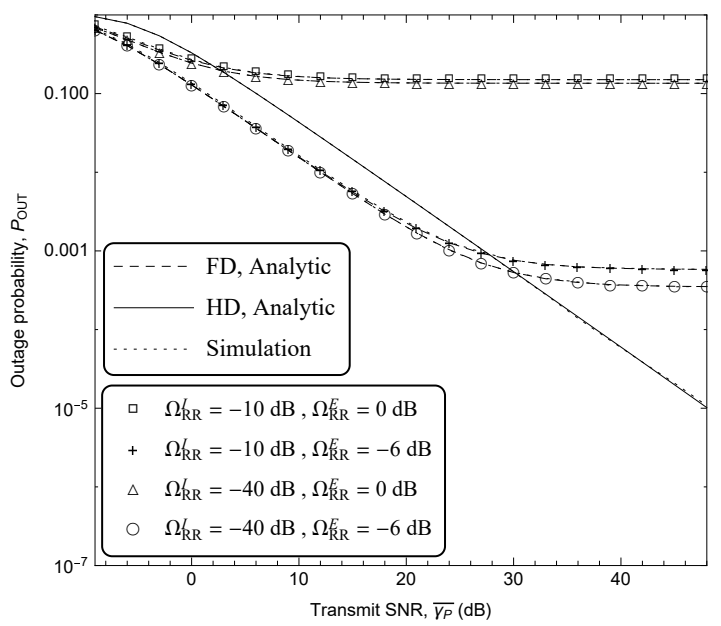

Fig. 5. Outage Probability versus Transmit SNR $\gamma_{P}$, for distinct values of average power at the RSIs links, $\Omega_{\mathrm{RR}}^{\mathrm{I}}$ and $\Omega_{\mathrm{RR}}^{\mathrm{E}}$, considering $\rho=0.5$
In order to complement the previous observations, Fig. 6 shows the outage probability as a function of the power splitting factor $\rho$, for different combinations of the RSI links, $\Omega_{\mathrm{RR}}^{\mathrm{I}}$ and $\Omega_{\mathrm{RR}}^{\mathrm{E}}$ and considering $\gamma_{P}=30 \mathrm{~dB}$. As previously observed in Fig. 5, reducing the average gain of the self-energy recycling channel is beneficial to the system performance. Notice that the self-energy recycling channel impacts on the optimal power splitting ratio value, that can be explained by the relation between both parameters in (6) and (7), given the DF protocol . Also notice that, in HD mode, the optimal power splitting ratio is closer to $\rho=0.5$ and, on the other hand, in the FD scenario, the system presents better results for lower values of $\rho<0.5$. In Addition, note that for higher values of $\rho$, there is a loss on the performance of FD cases due to increasing of the RSI. Therefore, as more power is dedicated to the information decoding process on the relay, FD will present better results compared to the HD-based system.

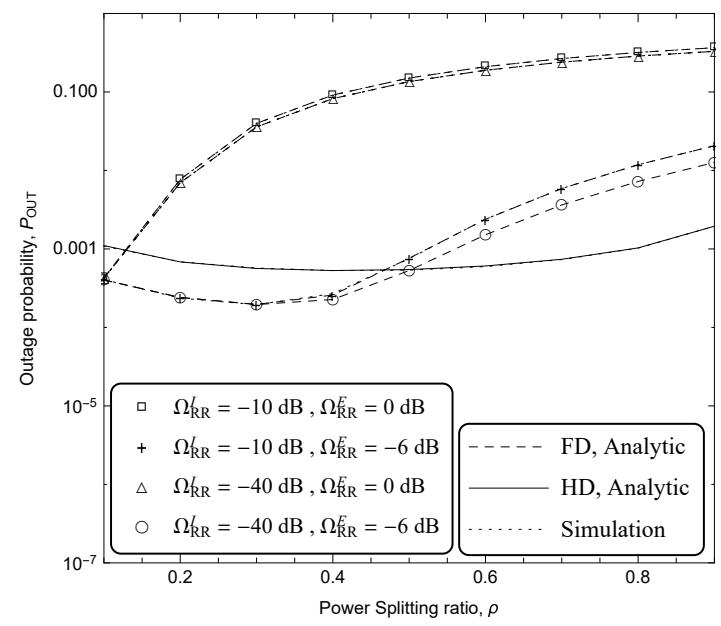

Fig. 6. Outage Probability versus power splitting factor $\rho$,for distinct values of average power at the RSIs links, $\Omega_{\mathrm{RR}}^{\mathrm{I}}$ and $\Omega_{\mathrm{RR}}^{\mathrm{E}}$, considering $\gamma_{P}=30 \mathrm{~dB}$.

Fig. 7 illustrates the outage probability versus the normalized relay position for $\rho=0.1,0.3,0.5,0.7$ considering full and half duplex mode at R. Beyond that, similar to other figures, Monte Carlo simulations are provided to validated the expressions. Notice that, excluding the cases $\rho=0.5$ and $\rho=0.7$ for FD, all scenarios present the best performance near the source, as expected. Also notice that the worst case happens when in FD mode, the power splitting ratio is equal to 0.7 . 


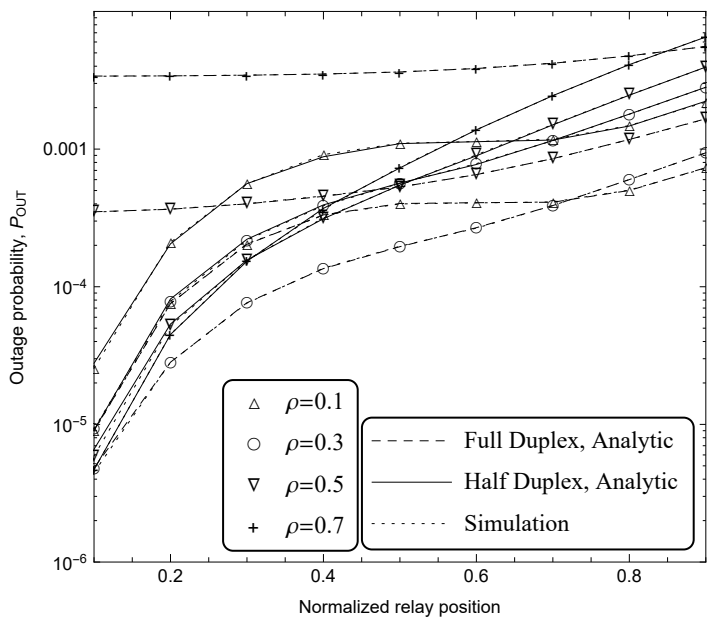

Fig. 7. Outage probability versus relative position of the relay, for $\rho=$ $0.1,0.3,0.5,0.7$ with $\gamma_{P}=30 \mathrm{~dB}$.

\section{CONCLUSiON}

In this work, the outage probability of a DF relaying network with an energy-constrained relay, where a PS-based SWIPT technique was used to provide the relay with energy for the retransmission process was studied. We derive tight approximated closed-form expressions for the outage probability of the considered FD case as well as for the HD case. Our analytical expressions were corroborated via Monte Carlos simulations. The results showed that the FDR network achieved better performance than the HDR network for lower values of the transmitted SNR due to the RSI impact on the performance of FD-based schemes. Furthermore, the increase on value of the average self-energy recycling channel impacts negatively on the performance of the system, thus FD mode overcomes HD mode when the relay dedicates more energy for the information decodification process, and the self-energy recycling remains low.

\section{ACKNOWLEDGMENTS}

This research has been partially supported by the São Paulo Research Foundation (FAPESP), Proc. 2019/23576-1, by the Academy of Finland 6Genesis Flagship under Grant 318927, EE-IoT under Grant 319008), Aka Prof under Grant 307492, and by the Brazilian National Council for Scientific and Technological Development (CNPq) under Grant 421850/2018-3.

\section{REFERENCES}

[1] ITU-R, "ITU-R M.[IMT-2020.TECH PERF REQ] - minimum requirements related to technical performance for imt-2020 radio interface(s)," Report ITU-R M.2410-0, Nov. 2017.

[2] N. H. Mahmood, H. Alves, O. A. López, M. Shehab, D. P. M. Osorio, and M. Latva-Aho, "Six key features of machine type communication in 6g," in 2020 2nd 6G Wireless Summit (6G SUMMIT), 2020, pp. 1-5.

[3] M. Agiwal, A. Roy, and N. Saxena, "Next generation 5g wireless networks: A comprehensive survey," IEEE Communications Surveys Tutorials, vol. 18, no. 3, pp. 1617-1655, thirdquarter 2016.

[4] M. Ku, W. Li, Y. Chen, and K. J. R. Liu, "Advances in energy harvesting communications: Past, present, and future challenges," IEEE Communications Surveys Tutorials, vol. 18, no. 2, pp. 1384-1412, Secondquarter 2016 .
[5] N. Zhao, S. Zhang, F. R. Yu, Y. Chen, A. Nallanathan, and V. C. M. Leung, "Exploiting interference for energy harvesting: A survey, research issues, and challenges," IEEE Access, vol. 5, pp. 10403-10421, 2017.

[6] T. D. P. Perera, D. N. K. Jayakody, S. K. Sharma, S. Chatzinotas, and J. Li, "Simultaneous wireless information and power transfer (swipt): Recent advances and future challenges," IEEE Communications Surveys Tutorials, vol. 20, no. 1, pp. 264-302, Firstquarter 2018.

[7] L. Liu, R. Zhang, and K. Chua, "Wireless information and power transfer: A dynamic power splitting approach," IEEE Transactions on Communications, vol. 61, no. 9, pp. 3990-4001, September 2013.

[8] Y. Liu, C. Yan, H. Yang, X. Bai, and L. Cong, "Optimal power splitting in wireless powered communication network with two-way relay," in 2017 IEEE 17th International Conference on Communication Technology (ICCT), Oct 2017, pp. 545-548.

[9] X. Zhou, R. Zhang, and C. K. Ho, "Wireless information and power transfer in multiuser ofdm systems," IEEE Transactions on Wireless Communications, vol. 13, no. 4, pp. 2282-2294, April 2014.

[10] I. Krikidis, S. Sasaki, S. Timotheou, and Z. Ding, "A low complexity antenna switching for joint wireless information and energy transfer in mimo relay channels," IEEE Transactions on Communications, vol. 62, no. 5, pp. 1577-1587, May 2014.

[11] C. Shen, W.-C. Li, and T.-H. Chang, "Simultaneous information and energy transfer: A two-user miso interference channel case," in 2012 IEEE Global Communications Conference (GLOBECOM), Dec 2012, pp. 3862-3867.

[12] Y. Zhang, J. He, S. Guo, and F. Wang, "Energy efficiency maximisation in wireless powered networks with cooperative non-orthogonal multiple access," IET Communications, vol. 12, no. 18, pp. 2374-2383, 2018.

[13] R. Zhang and C. K. Ho, "Mimo broadcasting for simultaneous wireless information and power transfer," IEEE Transactions on Wireless Communications, vol. 12, no. 5, pp. 1989-2001, May 2013.

[14] J. Laneman, D. Tse, and G.Wornell, "Cooperative diversity in wireless networks: Efficient protocols and outage behavior," IEEE Trans. Inf. Theory, vol. 50, no. 12, pp. 3062-3080, Dez. 2004.

[15] E. N. Egashira, E. E. Benitez Olivo, D. P. Moya Osorio, and H. Alves, "Secrecy performance of untrustworthy af relay networks using cooperative jamming and swipt," in 2019 IEEE 30th Annual International Symposium on Personal, Indoor and Mobile Radio Communications (PIMRC), Sep. 2019, pp. 1-6.

[16] Y. Zeng and R. Zhang, "Full-duplex wireless-powered relay with selfenergy recycling," IEEE Wireless Communications Letters, vol. 4, no. 2, pp. 201-204, April 2015

[17] D. P. M. Osorio, E. E. B. Olivo, H. Alves, J. C. S. S. Filho, and M. Latva-aho, "Exploiting the direct link in full-duplex amplify-andforward relaying networks," IEEE Signal Processing Letters, vol. 22, no. 10, pp. 1766-1770, Oct 2015.

[18] Z. Hu, C. Yuan, and F. Gao, "Maximizing harvested energy for fullduplex swipt system with power splitting," IEEE Access, vol. 5, pp. 24975-24 987, 2017.

[19] C. Zhong, H. A. Suraweera, G. Zheng, I. Krikidis, and Z. Zhang, "Wireless information and power transfer with full duplex relaying," IEEE Transactions on Communications, vol. 62, no. 10, pp. 3447-3461, Oct 2014.

[20] C. D. Nwankwo, L. Zhang, A. Quddus, M. A. Imran, and R. Tafazolli, "A survey of self-interference management techniques for single frequency full duplex systems," IEEE Access, vol. 6, pp. 30 242-30 268, 2018.

[21] H. Liu, K. J. Kim, K. S. Kwak, and H. Vincent Poor, "Power splittingbased swipt with decode-and-forward full-duplex relaying," IEEE Transactions on Wireless Communications, vol. 15, no. 11, pp. 7561-7577, Nov 2016.

[22] E. E. Benitez Olivo, D. P. Moya Osorio, H. Alves, J. Candido Silveira Santos Filho, and M. Latva-Aho, "Cognitive full-duplex decode-andforward relaying networks with usable direct link and transmit-power constraints," IEEE Access, vol. 6, pp. 24 983-24 995, 2018.

[23] Wolfram Research Inc. (2001-2008) ExpIntegralE. [Online]. Available: https:functions.wolfram.com/ExpIntegralE

[24] I. S. Gradshteyn and I. M. Ryzhik, Table of integrals, series, and products, 7th ed. Elsevier/Academic Press, Amsterdam, 2007. 